\title{
NANOSCALE MOLECULAR DYNAMICS SIMULATON OF SHOCK COMPRESSION OF SILICON
}

\author{
I.I. Oleynik ${ }^{1}$, S.V. Zybin ${ }^{2}$, M. L. Elert ${ }^{3}$, and C. T. White ${ }^{4}$ \\ ${ }^{1}$ Department of Physics, University of South Florida, Tampa, FL 33620 \\ ${ }^{2}$ Materials and Process Simulation Center, California Institute of Technology, Pasadena, CA 91125 \\ ${ }^{3}$ Chemistry Department, U. S. Naval Academy, Annapolis, MD 21402 \\ ${ }^{4}$ Naval Research Laboratory, Washington, DC 20375
}

\begin{abstract}
We report results of molecular dynamics simulation of shock wave propagation in silicon in [100], [110], and [111] directions obtained using a classical environment-dependent interatomic potential (EDIP). Several regimes of materials response are classified as a function of shock wave intensity using the calculated shock Hugoniot. Shock wave structure in [100] and [111] directions exhibit usual evolution as a function of piston velocity. At piston velocities $1.25<v_{p}<2.75 \mathrm{~km} / \mathrm{s}$ the shock wave consists of a fast elastic precursor followed by a slower plastic front. At larger piston velocities the single overdriven plastic wave propagates through the crystal causing amorphization of Si. However, the [110] shock wave exhibits an anomalous materials response at intermediate piston velocities around $v_{p} \simeq 1.75 \mathrm{~km} / \mathrm{s}$ which is characterized by the absence of plastic deformations.
\end{abstract}

Keywords: Shock waves, molecular dynamics, silicon, interatomic potentials

PACS: $62.50 .+p$, 82.40.Fp, 81.30.Hd, 46.40.Cd

\section{INTRODUCTION}

Shock waves in solids provide a unique opportunity to study the fundamental physics and chemistry of matter at extreme pressures and temperatures. In spite of substantial theoretical and experimental efforts, a full understanding of shockinduced elastic and plastic responses and polymorphic phase transitions is still far from complete. These phenomena often occur at the nanometer size and picosecond time scales which makes molecular dynamics (MD) simulations an ideal tool for exploring nanoscale mechanisms of shock induced processes such as shock-induced plasticity, chemical reactions and phase transitions $[1,2]$.

Recently we have performed MD simulations of shock wave propagation in diamond in [110] and
[111] directions and discovered a rich variety of materials response [3]. As the shock wave intensity increased, four different regimes of shock wave propagation were observed: (i) pure elastic wave, (ii) shock wave splitting into elastic and plastic waves, (iii) anomalous elastic regime, and (iv) overdriven plastic wave with carbon activated chemistry. The anomalous elastic response is characterized by the absence of plastic deformations: the material remains uniaxially compressed in spite of the substantial amount of shear stresses present behind the shock wave front. However, shear stresses developed at smaller piston velocities were substantially relieved by developing plastic deformations in the crystal. The effective freezing of plastic deformations was related to non-monotonic behavior of shear stresses 
upon uniaxial compression of diamond along both [110] and [111] directions.

In this regards, one might ask several important questions. Is this anomalous materials response a unique feature of diamond? Would other materials with non-monotonic dependence of shear stresses on uniaxial strain also exhibit similar effective freezing of plastic deformations at intermediate shock wave intensities? An obvious candidate to test this hypothesis is silicon. It is known that this covalently-bonded material has non-monotonic dependence of shear stresses on uniaxial strain [4]. Moreover, a substantial delay of plastic deformations has been recently observed in nanocrystalline silicon subjected to laser-induced shock compression [5]. Therefore, we decided to perform nanoscale MD simulations of shock wave propagation in silicon to address this scientifically interesting and conceptually challenging question.

\section{COMPUTATIONAL DETAILS}

The MD simulations of shock wave compression of silicon have been performed using the environment dependent interatomic potential (EDIP) [6]. EDIP includes environment-dependent changes in the bonding environment based on local coordination. It has been used extensively in simulations of extended defects in crystalline silicon and amorphous phases [7] and is considered to be one of the best silicon interatomic potentials currently available for MD simulations.

The MD shock wave simulations were set up by moving the diamond crystal at velocity $-u_{p}$ toward a fixed infinitely massive piston represented by a repulsive potential wall. Periodic boundary conditions were applied in the transverse $x$ and $y$ directions and the longitudinal $z$-axis was oriented along a particular ([100], [110] or [111]) crystallographic direction. After the crystal collides with the piston, the resulting shock wave propagates back into the crystal. The local variables such as temperature, stresses, mass velocity, density, and local potential energy are then calculated by spatial averaging over the grid of the averaging bins in $z$-direction. The MD simulations were performed in the range of piston velocities from 1 to $4 \mathrm{~km} / \mathrm{s}$.

\section{CLASSIFICATION OF MATERIALS RESPONSE USING SHOCK HUGONIOT}

Different regimes of materials response to shock compression are conveniently classified using the shock Hugoniot. In the case of silicon, they are elastic, plastic, 'anomalous elastic' and amorphization or activated silicon chemistry regimes, see Figure 1. The silicon [100], [110], and [111] shock Hugoniots were produced by processing results of molecular dynamics simulations. They serve as effective "phase diagrams" for the shock compressed material because different regimes of materials response are easily attributed to distinctive features of the shock Hugoniot.

In particular, at small piston velocities -alastic

plastic/amorphitization
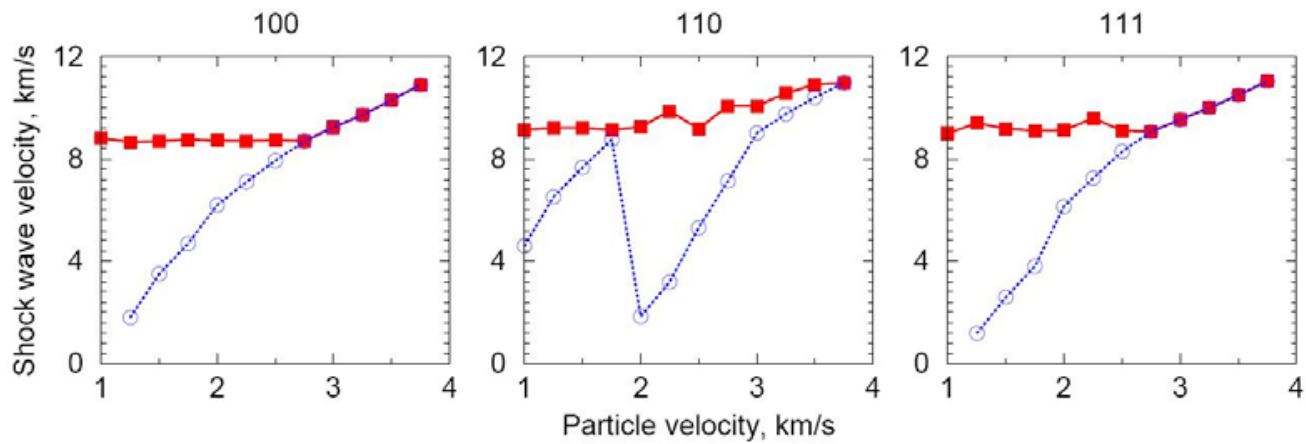

FIGURE 1. Silicon Hugoniot relationships between shock velocity and piston (particle) velocity for three crystallographic directions of shock wave propagation: [100], [110], [111] obtained from MD simulations. 

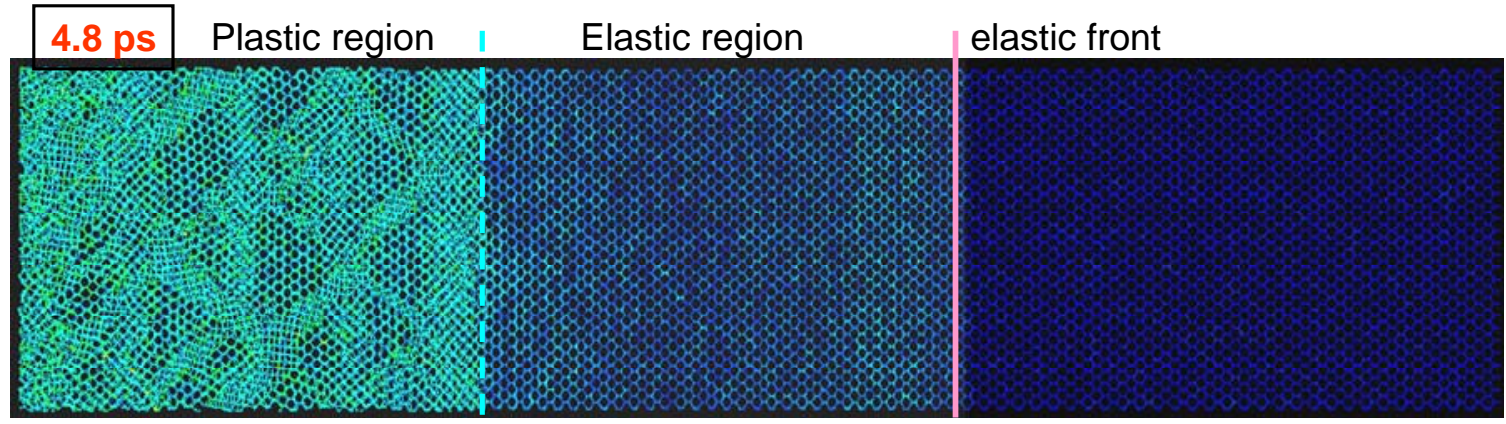

FIGURE 2. Snapshot of [100] two-wave shock splitting in silicon for piston velocity $1.75 \mathrm{~km} / \mathrm{s}$. Atoms are colored according to their potential energy.

$1.25<v_{p}<2.75 \mathrm{~km} / \mathrm{s}$ the [100] and [111] shock waves consist of an elastic precursor followed by a slower plastic deformation front. These two different states of compression correspond to two branches of shock Hugoniot, see Figure 1. Taking a sample of silicon, shock compressed at a particular value of piston velocity, one can examine its atomic structure. For example in the regime of elastic-plastic split-shock-wave one can clearly see the three states of the crystal: uncompressed in front of the shock wave front, elastically compressed behind the elastic shock wave front, and the part affected by plastic deformations taking place after the second plastic shock wave front. Figure 2 clearly shows these three states of the crystal in case of piston velocity $v_{p}=1.75 \mathrm{~km} / \mathrm{s}$.

At larger piston velocities, two branches of shock Hugoniot come together at the piston velocity corresponding to the onset of a single, overdriven plastic wave propagating through the crystal. In the case of silicon samples oriented along [100] and [111] directions this critical piston velocity is $v_{p}=2.75 \mathrm{~km} / \mathrm{s}$. As is seen from Figure 3 that shows the atomic structure of [100] shock compressed silicon at $v_{p}=3.00 \mathrm{~km} / \mathrm{s}$, a single overdriven plastic (amorphization) wave propagates through the crystal.

In contrast to [100] and [111] cases, the shock Hugoniot for a shock wave propagating in the [110] crystallographic direction clearly exhibits anomaly. At piston velocity $1.75 \mathrm{~km} / \mathrm{s}$, the elastic and plastic shock wave front coalesce into a single structure. It would be natural to assume that this piston velocity would produce a single, overdriven plastic wave as it was observed for the other, [100] and [111] directions. However, inspection of shock wave structure in Figure 4 reveals an absence of any plastic deformations, i.e. the crystal exhibits

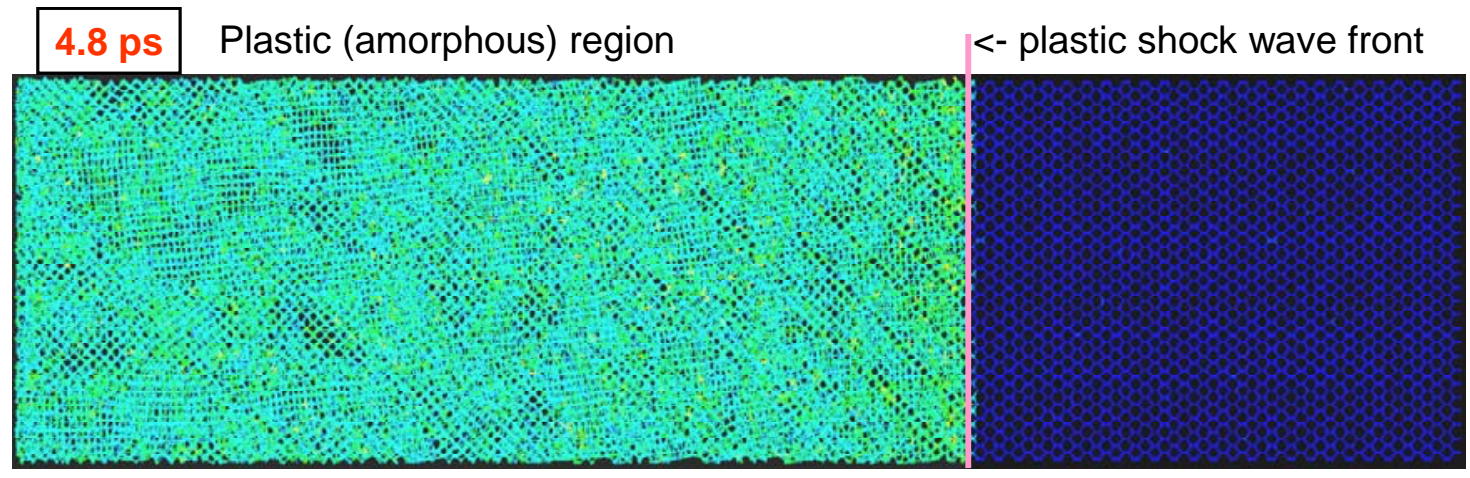

FIGURE 3. Snapshot of [100] single overdriven plastic/amorphization wave in silicon for piston velocity $3.0 \mathrm{~km} / \mathrm{s}$. Atoms are colored according to their potential energy. 

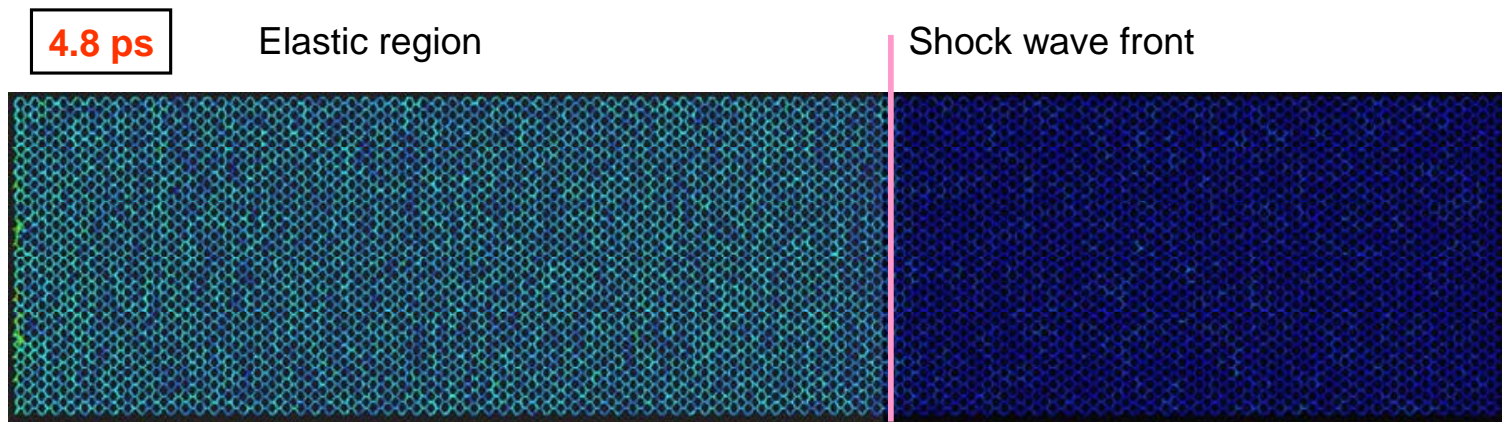

FIGURE 4. Snapshot of "anomalous elastic” shock wave propagating in [110] crystallographic direction initiated by piston with the velocity $1.75 \mathrm{~km} / \mathrm{s}$. Atoms are colored according to their potential energy.

"anomalous elastic" response in a sense that at smaller shock intensities, plastic deformations were present in the material.

Comparing to shock compression in diamond where an "anomalous elastic" wave was observed within a substantial interval of piston velocities for both [110] and [111] samples, the "anomalous elastic" wave in silicon appears only in the [110] sample and in a very narrow interval piston of velocities around $v_{p}=1.75 \mathrm{~km} / \mathrm{s}$. For the [100] and [111] directions, we did not find any cases of "anomalous elastic" response. This observation is related to the fact that diamond and silicon have different character of chemical bonding. The C-C bonds in diamond are very strong which results in relative stability of diamond phase and its resistance to chemical reactions and phase transformations at high pressures and temperatures. In contrast, silicon is relatively soft material that has several phase transitions occurring at relatively low pressures and room temperature. Therefore, the Si-Si bonds are easily broken and the pure "anomalous elastic" regime is difficult to sustain in a wide range of piston velocities.

The phenomenon of anomalous elastic response of the lattice upon strong shock compression has already been observed in several MD simulations including fcc rare gas solids [8], diamond [3], copper and aluminum [9]. All these materials exhibit non-monotonic shear-stress - uniaxial strain dependence. It is known that the shear stresses are the driving forces of plastic deformations in crystals. Therefore, the appearance of the 'anomalous elastic' regime in shock compressed material is directly related to nonmonotonic shear stress behavior upon uniaxial compression. We are performing a detailed investigation of shock compressed silicon with the aim to elucidate this relationship and provide a simple physical explanation of the anomalous elastic materials response upon shock compression.

\section{ACKNOWLEDGEMENTS}

The work at USF is supported by NSF-NIRT (ECS-0404137) and ARO-MURI (W901 1NF-051-0266). Funding at Caltech was provided by ONR and ARO-MURI. CTW is supported by ONR directly and through Naval Research Laboratory.

\section{REFERENCES}

1. Holian B.L., Shock Waves 13, 489 (2004).

2. Robertson D.H, Brenner D.W., and White C.T., Phys. Rev. Lett. 67, 3132 (1991).

3. Zybin S.Z., Oleynik I.I., Elert M.L., and White C.T., MRS Proceedings 800, AA7.7 (2003).

4. Swift D.C. and Ackland G.J., Appl. Phys. Lett. 83, 1151 (2003).

5. Loveridge-Smith A. et al., Phys. Rev. Lett. 86, 2349 (2001).

6. Bazant M.Z. and Kaxiras E., Phys. Rev. Lett. 77, 4370 (1996).

7. Justo J.F., Bazant M.Z., Kaxiras E., Bulatov V.V, and Yip S., Phys. Rev. B 58, 2539 (1998).

8. Zhakhovskii V.V., Zybin S.V., Nishihara K., and Anisimov S.I., Progr. Theor. Phys. Suppl. 138, 223 (2000).

9. Bringa E.M., to be published. 